\title{
Ginsparg-Wilson pions scattering in a sea of staggered quarks
}

\author{
Jiunn-Wei Chen, ${ }^{1, *}$ Donal O’Connell, ${ }^{2, \dagger}$ Ruth Van de Water, ${ }^{3, \ddagger}$ and André Walker-Loud ${ }^{3, \S}$ \\ ${ }^{1}$ Department of Physics, National Taiwan University, Taipei 10617, Taiwan \\ ${ }^{2}$ California Institute of Technology, Pasadena, California 91125, USA \\ ${ }^{3}$ Department of Physics, University of Washington, Box 351560, Seattle, Washington 98195-1560, USA
}

(Received 6 January 2006; revised manuscript received 20 March 2006; published 24 April 2006)

\begin{abstract}
We calculate isospin 2 pion-pion scattering in chiral perturbation theory for a partially quenched, mixed action theory with Ginsparg-Wilson valence quarks and staggered sea quarks. We point out that for some scattering channels, the power-law volume dependence of two-pion states in nonunitary theories such as partially quenched or mixed action QCD is identical to that of QCD. Thus one can extract infinite-volume scattering parameters from mixed action simulations. We then determine the scattering length for both 2 and $2+1$ sea quarks in the isospin limit. The scattering length, when expressed in terms of the pion mass and the decay constant measured on the lattice, has no contributions from mixed valence-sea mesons, thus it does not depend upon the parameter, $C_{\text {Mix }}$, that appears in the chiral Lagrangian of the mixed theory. In addition, the contributions which nominally arise from operators appearing in the mixed action $\mathcal{O}\left(a^{2} m_{q}\right)$ Lagrangian exactly cancel when the scattering length is written in this form. This is in contrast to the scattering length expressed in terms of the bare parameters of the chiral Lagrangian, which explicitly exhibits all the sicknesses and lattice spacing dependence allowed by a partially quenched mixed action theory. These results hold for both 2 and $2+1$ flavors of sea quarks.
\end{abstract}

DOI: 10.1103/PhysRevD.73.074510

PACS numbers: $12.38 . G c$

\section{INTRODUCTION}

Lattice QCD can, in principle, be used to calculate precisely low energy quantities including hadron masses, decay constants, and form factors. In practice, however, limited computing resources make it currently impossible to calculate processes with dynamical quark masses as light as those in the real world. Thus one performs simulations with quark masses that are as light as possible and then extrapolates the lattice calculations to the physical values using expressions calculated in chiral perturbation theory $(\chi \mathrm{PT})$. This, of course, relies on the assumption that the quark masses are light enough that one is in the chiral regime and can trust $\chi \mathrm{PT}$ to be a good effective theory of QCD [1,2].

Lattice simulations with staggered fermions [3] can at present reach significantly lighter quark masses than other fermion discretizations and have proven extremely successful in accurately reproducing experimentally measurable quantities $[4,5]$. Staggered fermions, however, have the disadvantage that each quark flavor comes in four tastes. Because these species are degenerate in the continuum, one can formally remove them by taking the fourth root of the quark determinant. In practice, however, the fourth root must be taken before the continuum limit; thus it is an open theoretical question whether or not this fourth-

\footnotetext{
*Electronic address: jwc@ @ phys.ntu.edu.tw

${ }^{\dagger}$ Electronic address: donal@ theory.caltech.edu

Electronic address: ruthv@fnal.gov

${ }^{\S}$ Electronic address: walkloud@u.washington.edu
}

rooted theory becomes QCD in the continuum limit. ${ }^{1}$ Even if one assumes the validity of the fourth-root trick, which we do in the rest of this paper, staggered fermions have other drawbacks. On the lattice, the four tastes of each quark flavor are no longer degenerate, and this taste symmetry breaking is numerically significant in current simulations [5]. Thus one must use staggered chiral perturbation theory $(\mathrm{S} \chi \mathrm{PT})$, which accounts for taste-breaking discretization effects, to extrapolate correctly staggered lattice calculations to the continuum [7-10]. Fits of $\mathrm{S} \chi \mathrm{PT}$ expressions for meson masses and decay constants have been remarkably successful. Nevertheless, the large number of operators in the next-to-leading order (NLO) staggered chiral Lagrangian [10] and the complicated form of the kaon $B$-parameter in $\mathrm{S} \chi \mathrm{PT}$ [11] both show that $\mathrm{S} \chi \mathrm{PT}$ expressions for many physical quantities will contain a daunting number of undetermined fit parameters. Another practical hindrance to the use of staggered fermions as valence quarks is the construction of lattice interpolating fields. Although the construction of a staggered interpolating field is straightforward for mesons since they are spin 0 objects [12,13], this is not in general the case for vector mesons, baryons or multihadron states since the lattice rotation operators mix the spin, angular momentum and taste of a given interpolating field [14-16].

The use of Ginsparg-Wilson (GW) fermions [17] evades both the practical and theoretical issues associated with

\footnotetext{
${ }^{1}$ See Ref. [6] for a recent review of staggered fermions and the fourth-root trick.
} 
staggered fermions. Because GW fermions are tasteless, one can simply construct interpolating operators with the right quantum numbers for the desired meson or baryon. Moreover, massless GW fermions possess an exact chiral symmetry on the lattice [18] which protects expressions in $\chi$ PT from becoming unwieldy. ${ }^{2}$ Unfortunately, simulations with dynamical GW quarks are approximately 10 to 100 times slower than those with staggered quarks [25] and thus are not presently practical for realizing light quark masses.

A practical compromise is therefore the use of $\mathrm{GW}$ valence quarks and staggered sea quarks. This so-called "mixed action" theory is particularly appealing because the MILC improved staggered field configurations are publicly available. Thus one only needs to calculate correlation functions on top of these background configurations, making the numerical cost comparable to that of quenched GW simulations. Several lattice calculations using domain-wall or overlap valence quarks with the MILC configurations are underway [26-28], including a determination of the isospin $2(I=2) \pi \pi$ scattering length [29]. Although this is not the first $I=2 \pi \pi$ scattering lattice simulation [30-34], it is the only one with pions light enough to be in the chiral regime [1,2]. Its precision is limited, however, without the appropriate mixed action $\chi \mathrm{PT}$ expression for use in continuum and chiral extrapolation of the lattice data. With this motivation we calculate the $I=2 \pi \pi$ scattering length in chiral perturbation theory for a mixed action theory with GW valence quarks and staggered sea quarks.

Mixed action chiral perturbation theory (MA $\chi \mathrm{PT})$ was first introduced in Refs. [35-37] and was extended to include GW valence quarks on staggered sea quarks for both mesons and baryons in Refs. [38,39], respectively. $\pi \pi$ scattering is well understood in continuum, infinite-volume $\chi \mathrm{PT}[40-46]$, and is the simplest two-hadron process that one can study numerically with LQCD. We extend the NLO $\chi$ PT calculations of Refs. [41,42] to MA $\chi$ PT. A mixed action simulation necessarily involves partially quenched QCD (PQQCD) [47-52], in which the valence and sea quarks are treated differently. Consequently, we provide the $\mathrm{PQ} \chi \mathrm{PT} \pi \pi$ scattering amplitude by taking an appropriate limit of our MA $\chi \mathrm{PT}$ expressions. In all of our computations, we work in the isospin limit both in the sea and valence sectors.

This article is organized as follows. We first comment on the determination of infinite-volume scattering parameters from lattice simulations in Sec. II, focusing on the applicability of Lüscher's method $[53,54]$ to mixed action lattice simulations. We then review mixed action LQCD and $\mathrm{MA} \chi \mathrm{PT}$ in Sec. III. In Sec. IV we calculate the $I=2$

\footnotetext{
${ }^{2}$ In practice, the degree of chiral symmetry is limited by how well the domain-wall fermion [19-21] is realized or the overlap operator [22-24] is approximated.
}

$\pi \pi$ scattering amplitude in $\mathrm{MA} \chi \mathrm{PT}$, first by reviewing $\pi \pi$ scattering in continuum $S U(2) \chi \mathrm{PT}$ and then by extending to partially quenched mixed action theories with $N_{f}=2$ and $N_{f}=2+1$ sea quarks. We discuss the role of the double poles in this process [55] and parameterize the partial quenching effects in a particularly useful way for taking various interesting and important limits. Next, in Sec. V, we present results for the pion scattering length in both 2 and $2+1$ flavor MA $\chi$ PT. These expressions show that it is advantageous to fit to partially quenched lattice data using the lattice pion mass and pion decay constant measured on the lattice rather than the LO parameters in the chiral Lagrangian. We also give expressions for the corresponding continuum $\mathrm{PQ} \chi \mathrm{PT}$ scattering amplitudes, which do not already appear in the literature. Finally, in Sec. VI we briefly discuss how to use our MA $\chi$ PT formulae to determine the physical scattering length in QCD from mixed action lattice data and conclude.

\section{DETERMINATION OF SCATTERING PARAMETERS FROM MIXED ACTION LATTICE SIMULATIONS}

Lattice QCD calculations are performed in Euclidean spacetime, thereby precluding the extraction of $S$-matrix elements from infinite volume [56]. Lüscher, however, developed a method to extract the scattering phase shifts of two-particle scattering states in quantum field theory by studying the volume dependence of two-point correlation functions in Euclidean spacetime [53,54]. In particular, for two particles of equal mass $m$ in an $s$-wave state with zero total 3-momentum in a finite volume, the difference between the energy of the two particles and twice their rest mass is related to the $s$-wave scattering length ${ }^{3}$ :

$$
\Delta E_{0}=-\frac{4 \pi a_{0}}{m \mathrm{~L}^{3}}\left[1+c_{1} \frac{a_{0}}{\mathrm{~L}}+c_{2}\left(\frac{a_{0}}{\mathrm{~L}}\right)^{2}+\mathcal{O}\left(\frac{1}{\mathrm{~L}^{3}}\right)\right] .
$$

In the above expression, $a_{0}$ is the scattering length (not to be confused with the lattice spacing, $a$ ), $\mathrm{L}$ is the length of one side of the spatially symmetric lattice, and $c_{1}$ and $c_{2}$ are known geometric coefficients. ${ }^{4}$ Thus, even though one cannot directly calculate scattering amplitudes with lattice simulations, Eq. (1), which we will refer to as Lüscher's formula, allows one to determine the infinite-volume scattering length. One can then use the expression for the scattering length computed in infinite volume $\chi \mathrm{PT}$ to extrapolate the lattice data to the physical quark masses.

Because Lüscher's method requires the extraction of energy levels, it relies upon the existence of a Hamil-

\footnotetext{
${ }^{3}$ Here we use the "particle physics" definition of the scattering length which is opposite in sign to the "nuclear physics" definition.

${ }^{4}$ This expression generalizes to scattering parameters of higher partial waves and nonstationary particles [53,54,57,58].
} 
tonian for the theory being studied. This has not been demonstrated (and is likely false) for partially quenched and mixed action QCD, both of which are nonunitary. Nevertheless, one can calculate the ratio of the two-pion correlator to the square of the single-pion correlator in lattice simulations of these theories and extract the coefficient of the term which is linear in time, which becomes the energy shift in the QCD (and continuum) limit. We claim that in certain scattering channels, despite the inherent sicknesses of partially quenched and mixed action QCD, this quantity is still related to the infinite-volume scattering length via Eq. (1), i.e. the volume dependence is identical to Eq. (1) up to exponentially suppressed corrections. ${ }^{5}$ This is what we mean by "Lüscher's method" for nonunitary theories. We will expand upon this point in the following paragraphs.

It is well known that Lüscher's formula does not hold for many scattering channels in quenched theories because unitarity-violating diagrams give rise to enhanced finite volume effects [59]. For certain scattering channels, however, quenched $\chi \mathrm{PT}$ calculations in finite volume show that, at 1-loop order, the volume dependence is identical in form to Lüscher's formula [59-61]. Chiral perturbation theory calculations additionally show that the same sicknesses that generate enhanced finite volume effects in quenched QCD also do so in partially quenched and mixed action theories [35,36,38,50,51,62-64]. It then follows that if a given scattering channel has the same volume dependence as Eq. (1) in quenched QCD, the corresponding partially quenched (and mixed action) two-particle process will also obey Eq. (1). Correspondingly, scattering channels which have enhanced volume dependence in quenched QCD also have enhanced volume dependence in partially quenched and mixed action theories. We now proceed to discuss in some detail why Lüscher's formula does or does not hold for various $2 \rightarrow 2$ scattering channels.

Finite volume effects in lattice simulations come from the ability of particles to propagate over long distances and feel the finite extent of the box through boundary conditions. Generically, they are proportional either to inverse powers of $\mathrm{L}$ or to $\exp (-m \mathrm{~L})$, but Lüscher's formula neglects exponentially suppressed corrections. Calculations of scattering processes in effective field theories at finite volume show that the power-law corrections only arise from $s$-channel diagrams $[59,61,62,65,66]$. This is because all of the intermediate particles can go on-shell simultaneously, and thus are most sensitive to boundary effects. Consequently, when there are no unitarity-violating effects in the $s$-channel diagrams for a particular scattering process, the volume dependence will be identical to Eq. (1), up to exponential corrections. Unitarity-violating hairpin

\footnotetext{
${ }^{5}$ Here, and in the following discussion, we restrict ourselves to a perturbative analysis.
}

propagators in $s$-channel diagrams, however, give rise to enhanced volume corrections because they contain double poles which are more sensitive to boundary effects [59]. ${ }^{6}$ Thus all violations of Lüscher's formula come from onshell hairpins in the $s$-channel.

Let us now consider $I=2 \pi \pi$ scattering in the mixed action theory. All intermediate states must have isospin 2 and $s \geq 4 \mathrm{~m}^{2}$. If one cuts an arbitrary graph connecting the incoming and outgoing pions, there is only enough energy for two of the internal pions to be on-shell, and, by conservation of isospin, they must be valence $\pi^{+}$'s. ${ }^{7}$ Thus no hairpin diagrams ever go on-shell in the $s$-channel, and the structure of the integrals which contribute to the power-law volume dependence in the partially quenched and mixed action theories is identical to that in continuum $\chi \mathrm{PT}$. This insures that Lüscher's formula is correctly reproduced to all orders in $1 / \mathrm{L}$ with the correct ratios between coefficients of the various terms. Moreover, this holds to all orders in $\chi \mathrm{PT}, \mathrm{PQ} \chi \mathrm{PT}, \mathrm{MA} \chi \mathrm{PT}$, and even quenched $\chi \mathrm{PT}$. The sicknesses of the partially quenched and mixed action theories only alter the exponential volume dependence of the $I=2$ scattering amplitude. ${ }^{8}$ This is in contrast to the $I=0 \pi \pi$ amplitude, which suffers from enhanced volume corrections away from the QCD limit. In general, the argument which protects Lüscher's formula from enhanced powerlike volume corrections holds for all "maximally-stretched" states at threshold in the meson sector, i.e. those with the maximal values of all conserved quantum numbers; other examples include $K^{+} K^{+}$ and $K^{+} \pi^{+}$scattering. We expect that a similar argument will hold for certain scattering channels in the baryon sector.

Therefore the $s$-wave $I=2 \pi \pi$ scattering length can be extracted from mixed action lattice simulations using Lüscher's formula and then extrapolated to the physical quark masses and to the continuum using the infinite-volume MA $\chi \mathrm{PT}$ expression for the scattering length. ${ }^{9}$

\footnotetext{
${ }^{6} \mathrm{We}$ note that, while the enhanced volume corrections in quenched QCD invalidate the extraction of scattering parameters from certain scattering channels, e.g. $I=0[59,61]$, this is not the case in principle for partially quenched QCD, since QCD is a subset of the theory. Because the enhanced volume contributions must vanish in the QCD limit, they provide a "handle" on the enhanced volume terms. In practice, however, these enhanced volume terms may dominate the correlation function, making the extraction of the desired (nonenhanced) volume dependence impractical.

${ }^{7}$ We restrict the incoming pions to be below the inelastic threshold; this is necessary for the validity of Lüscher's formula even in QCD.

${ }^{8}$ In fact, hairpin propagators will give larger exponential dependence than standard propagators because they are more chirally sensitive.

${ }^{9}$ For a related discussion, see Ref. [67].
} 


\section{MIXED ACTION LAGRANGIAN AND PARTIAL QUENCHING}

Mixed action theories use different discretization techniques in the valence and sea sectors and are therefore a natural extension of partially quenched theories. We consider a theory with $N_{f}$ staggered sea quarks and $N_{v}$ valence quarks (with $N_{v}$ corresponding ghost quarks) which satisfy the Ginsparg-Wilson relation $[17,18]$. In particular we are interested in theories with two light dynamical quarks $\left(N_{f}=2\right)$ and with three dynamical quarks where the two light quarks are degenerate (commonly referred to as $N_{f}=$ $2+1)$. To construct the continuum effective Lagrangian which includes lattice artifacts one follows the two step procedure outlined in Ref. [68]. First one constructs the Symanzik continuum effective Lagrangian at the quark level $[69,70]$ up to a given order in the lattice spacing, $a$ :

$$
\mathcal{L}_{\text {Sym }}=\mathcal{L}+a \mathcal{L}^{(5)}+a^{2} \mathcal{L}^{(6)}+\ldots,
$$

where $\mathcal{L}^{(4+n)}$ contains higher dimensional operators of dimension $4+n$. Next one uses the method of spurion analysis to map the Symanzik action onto a chiral Lagrangian, in terms of pseudo-Goldstone mesons, which now incorporates the lattice spacing effects. This has been done in detail for a mixed GW-staggered theory in Ref. [38]; here we only describe the results.

The leading quark level Lagrangian is given by

$$
\mathcal{L}=\sum_{a, b=1}^{4 N_{f}+2 N_{v}} \bar{Q}^{a}\left[i \not D-m_{Q}\right]_{a}{ }^{b} Q_{b},
$$

where the quark fields are collected in the vectors

$$
\begin{gathered}
Q^{N_{f}=2}=(\underbrace{u, d}_{\text {valence }}, \underbrace{j_{1}, j_{2}, j_{3}, j_{4}, l_{1}, l_{2}, l_{3}, l_{4}}_{\text {sea }}, \underbrace{\tilde{u}, \tilde{d}}_{\text {ghost }})^{T}, \\
Q^{N_{f}=2+1}=(\underbrace{u, d, s}_{\text {valence }} \underbrace{j_{1}, j_{2}, j_{3}, j_{4}, l_{1}, l_{2}, l_{3}, l_{4}, r_{1}, r_{2}, r_{3}, r_{4}}_{\text {sea }}, \underbrace{\tilde{u}, \tilde{d}, \tilde{s}}_{\text {ghost }})^{T}
\end{gathered}
$$

for the two theories. There are 4 tastes for each flavor of sea quark, $j, l, r .{ }^{10}$ We work in the isospin limit in both the valence and sea sectors so the quark mass matrix in the $2+1$ sea flavor theory is given by

$$
m_{Q}=\operatorname{diag}(\underbrace{m_{u}, m_{u}, m_{s}}_{\text {valence }}, \underbrace{m_{j}, m_{j}, m_{j}, m_{j}, m_{j}, m_{j}, m_{j}, m_{j}, m_{r}, m_{r}, m_{r}, m_{r}}_{\text {sea }}, \underbrace{m_{u}, m_{u}, m_{s}}_{\text {ghost }}) .
$$

The quark mass matrix in the two flavor theory is analogous but without strange valence, sea and ghost quark masses. The leading order mixed action Lagrangian, Eq. (3), has an approximate graded chiral symmetry, $S U\left(4 N_{f}+N_{v} \mid N_{v}\right)_{L} \otimes S U\left(4 N_{f}+N_{v} \mid N_{v}\right)_{R}$, which is exact in the massless limit. ${ }^{11}$ In analogy to QCD, we assume that the vacuum spontaneously breaks this symmetry down to its vector subgroup, $S U\left(4 N_{f}+N_{v} \mid N_{v}\right)_{V}$, giving rise to $\left(4 N_{f}+2 N_{v}\right)^{2}-1$ pseudo-Goldstone mesons. These mesons are contained in the field

$$
\Sigma=\exp \left(\frac{2 i \Phi}{f}\right), \quad \Phi=\left(\begin{array}{cc}
M & \chi^{\dagger} \\
\chi & \tilde{M}
\end{array}\right)
$$

The matrices $M$ and $\tilde{M}$ contain bosonic mesons while $\chi$ and $\chi^{\dagger}$ contain fermionic mesons. Specifically,

\footnotetext{
${ }^{10}$ Note that we use different labels for the valence and sea quarks than Ref. [38]. Instead we use the "nuclear physics" labeling convention, which is consistent with Ref. [39].

${ }^{11}$ This is a "fake" symmetry of PQQCD. However, it gives the correct Ward identities and thus can be used to understand the symmetries and symmetry breaking of PQQCD [51].
}

$$
\begin{aligned}
M & =\left(\begin{array}{cccccc}
\eta_{u} & \pi^{+} & \ldots & \phi_{u j} & \phi_{u l} & \ldots \\
\pi^{-} & \eta_{d} & \ldots & \phi_{d j} & \phi_{d l} & \ldots \\
\vdots & \vdots & \ddots & \ldots & \ldots & \ldots \\
\phi_{j u} & \phi_{j d} & \vdots & \eta_{j} & \phi_{j l} & \ldots \\
\phi_{l u} & \phi_{l d} & \vdots & \phi_{l j} & \eta_{l} & \ldots \\
\vdots & \vdots & \vdots & \vdots & \vdots & \ddots
\end{array}\right), \\
\tilde{M} & =\left(\begin{array}{ccc}
\tilde{\eta}_{u} & \tilde{\pi}^{+} & \ldots \\
\tilde{\pi}^{-} & \tilde{\eta}_{d} & \ldots \\
\vdots & \vdots & \ddots
\end{array}\right) \\
\chi & =\left(\begin{array}{cccccc}
\phi_{\tilde{u} u} & \phi_{\tilde{u} d} & \ldots & \phi_{\tilde{u} j} & \phi_{\tilde{u} l} & \ldots \\
\phi_{\tilde{d} u} & \phi_{\tilde{d} d} & \ldots & \phi_{\tilde{d} j} & \phi_{\tilde{d} l} & \ldots \\
\vdots & \vdots & \vdots & \vdots & \vdots & \ddots
\end{array}\right) .
\end{aligned}
$$

In Eq. (8) we only explicitly show the mesons needed in the two flavor theory. The ellipses indicate mesons containing strange quarks in the $2+1$ theory. The upper $N_{v} \times N_{v}$ block of $M$ contains the usual mesons composed of a valence quark and antiquark. The fields composed of one 
valence quark and one sea antiquark, such as $\phi_{u j}$, are $1 \times 4$ matrices of fields where we have suppressed the taste index on the sea quarks. Likewise, the sea-sea mesons such as $\phi_{j l}$ are $4 \times 4$ matrix-fields. Under chiral transformations, $\Sigma$ transforms as

$$
\Sigma \rightarrow L \Sigma R^{\dagger}, \quad L, R \in S U\left(4 N_{f}+N_{v} \mid N_{v}\right)_{L, R} .
$$

In order to construct the chiral Lagrangian it is useful to first define a power-counting scheme. Continuum $\chi \mathrm{PT}$ is an expansion in powers of the pseudo-Goldstone meson momentum and mass squared [41,42]:

$$
\varepsilon^{2} \sim p_{\pi}^{2} / \Lambda_{\chi}^{2} \sim m_{\pi}^{2} / \Lambda_{\chi}^{2},
$$

where $m_{\pi}^{2} \propto m_{Q}$ and $\Lambda_{\chi}$ is the cutoff of $\chi \mathrm{PT}$. In a mixed theory (or any theory which incorporates lattice spacing artifacts) one must also include the lattice spacing in the power counting. Both the chiral symmetry of the GinspargWilson valence quarks and the remnant $U(1)_{A}$ symmetry of the staggered sea quarks forbid operators of dimension five; therefore the leading lattice spacing correction for this mixed action theory arises at $\mathcal{O}\left(a^{2}\right)$. Moreover, current staggered lattice simulations indicate that taste-breaking effects (which are of $\mathcal{O}\left(a^{2}\right)$ ) are numerically of the same size as the lightest staggered meson mass [5]. We therefore adopt the following power-counting scheme:

$$
\varepsilon^{2} \sim p_{\pi}^{2} / \Lambda_{\chi}^{2} \sim m_{Q} / \Lambda_{\mathrm{QCD}} \sim a^{2} \Lambda_{\mathrm{QCD}}^{2} .
$$

The leading order $(\mathrm{LO}), \mathcal{O}\left(\varepsilon^{2}\right)$, Lagrangian is then given in Minkowski space by [38]

$$
\begin{aligned}
\mathcal{L}= & \frac{f^{2}}{8} \operatorname{str}\left(\partial_{\mu} \Sigma \partial^{\mu} \Sigma^{\dagger}\right)+\frac{f^{2} B}{4} \operatorname{str}\left(\Sigma m_{Q}^{\dagger}+m_{Q} \Sigma^{\dagger}\right) \\
& -a^{2}\left(\mathcal{U}_{S}+\mathcal{U}_{S}^{\prime}+\mathcal{U}_{V}\right),
\end{aligned}
$$

where we use the normalization $f \sim 132 \mathrm{MeV}$ and have already integrated out the taste singlet $\Phi_{0}$ field, which is proportional to $\operatorname{str}(\Phi)$ [51]. $\mathcal{U}_{S}$ and $\mathcal{U}_{S}^{\prime}$ are the wellknown taste-breaking potential arising from the staggered sea quarks $[7,8]$. The staggered potential only enters into our calculation through an additive shift to the sea-sea meson masses; we therefore do not write out its explicit form. The enhanced chiral properties of the mixed action theory are illustrated by the fact that only one new potential term arises at this order:

$$
\mathcal{U}_{V}=-C_{\mathrm{Mix}} \operatorname{str}\left(\tau_{3} \Sigma \tau_{3} \Sigma^{\dagger}\right),
$$

where

$$
\tau_{3}=\mathcal{P}_{S}-\mathcal{P}_{V}=\operatorname{diag}\left(-I_{V}, I_{t} \otimes I_{S},-I_{V}\right) .
$$

The projectors, $\mathcal{P}_{S}$ and $\mathcal{P}_{V}$, project onto the sea and valence-ghost sectors of the theory, $I_{V}$ and $I_{S}$ are the valence and sea flavor identities, and $I_{t}$ is the taste identity matrix. From this Lagrangian, one can compute the LO masses of the various pseudo-Goldstone mesons in Eq. (8). For mesons composed of only valence (ghost) quarks of flavors $a$ and $b$,

$$
m_{a b}^{2}=B\left(m_{a}+m_{b}\right) .
$$

This is identical to the continuum LO meson mass because the chiral properties of Ginsparg-Wilson quarks protect mesons composed of only valence (ghost) quarks from receiving mass corrections proportional to the lattice spacing. However, mesons composed of only sea quarks of flavors $s_{1}$ and $s_{2}$ and taste $t$, or mixed mesons with one valence $(v)$ and one sea $(s)$ quark both receive lattice spacing mass shifts. Their LO masses are given by

$$
\begin{gathered}
\tilde{m}_{s_{1} s_{2}, t}^{2}=B\left(m_{s_{1}}+m_{s_{2}}\right)+a^{2} \Delta\left(\xi_{t}\right), \\
\tilde{m}_{v s}^{2}=B\left(m_{v}+m_{s}\right)+a^{2} \Delta_{\text {Mix }} .
\end{gathered}
$$

From now on we use tildes to indicate masses that include lattice spacing shifts. The only sea-sea mesons that enter $\pi \pi$ scattering to the order at which we are working are the taste-singlet mesons (this is because the valence-valence pions that are being scattered are tasteless), which are the heaviest; we therefore drop the taste label, $t$. The splittings between meson masses of different tastes have been determined numerically on the MILC configurations [5], so $\Delta\left(\xi_{I}\right)$ should be considered an input rather than a fit parameter. The mixed mesons all receive the same $a^{2}$ shift given by

$$
\Delta_{\mathrm{Mix}}=\frac{16 C_{\mathrm{Mix}}}{f^{2}},
$$

which has yet to be determined numerically.

After integrating out the $\Phi_{0}$ field, the two-point correlation functions for the flavor-neutral states deviate from the simple single pole form. The momentum space propagator between two flavor-neutral states is found to be at leading order [51]

$$
G_{\eta_{a} \eta_{b}}\left(p^{2}\right)=\frac{i \epsilon_{a} \delta_{a b}}{p^{2}-m_{\eta_{a}}^{2}+i \epsilon}-\frac{i}{N_{f}} \frac{\prod_{k=1}^{N_{f}}\left(p^{2}-\tilde{m}_{k}^{2}+i \epsilon\right)}{\left(p^{2}-m_{\eta_{a}}^{2}+i \epsilon\right)\left(p^{2}-m_{\eta_{b}}^{2}+i \epsilon\right) \prod_{k^{\prime}=1}^{N_{f}-1}\left(p^{2}-\tilde{m}_{k^{\prime}}^{2}+i \epsilon\right)},
$$

where

$$
\epsilon_{a}= \begin{cases}+1 & \text { for } a=\text { valence or sea quarks } \\ -1 & \text { for } a=\text { ghost quarks. }\end{cases}
$$

In Eq. (19), $k$ runs over the flavor-neutral states $\left(\phi_{j j}, \phi_{l l}, \phi_{r r}\right)$ and $k^{\prime}$ runs over the mass eigenstates of the sea sector. For $\pi \pi$ scattering, it will be useful to work with linear combinations of these $\eta_{a}$ fields. In par- 
ticular we form the linear combinations

$$
\pi^{0}=\frac{1}{\sqrt{2}}\left(\eta_{u}-\eta_{d}\right), \quad \bar{\eta}=\frac{1}{\sqrt{2}}\left(\eta_{u}+\eta_{d}\right),
$$

for which the propagators are

$$
\begin{gathered}
\mathcal{G}_{\pi^{0}}\left(p^{2}\right)=\frac{i}{p^{2}-m_{\pi}^{2}+i \epsilon}, \\
\mathcal{G}_{\bar{\eta}}\left(p^{2}\right)=\frac{i}{p^{2}-m_{\pi}^{2}+i \epsilon} \\
-\frac{2 i}{N_{f}} \frac{\prod_{k=1}^{N_{f}}\left(p^{2}-\tilde{m}_{k}^{2}+i \epsilon\right)}{\left(p^{2}-m_{\pi}^{2}+i \epsilon\right)^{2} \prod_{k^{\prime}=1}^{N_{f}-1}\left(p^{2}-\tilde{m}_{k^{\prime}}^{2}+i \epsilon\right)} .
\end{gathered}
$$

Specifically,

$$
\begin{gathered}
\mathcal{G}_{\bar{\eta}}\left(p^{2}\right)=\frac{i}{p^{2}-m_{\pi}^{2}}-i \frac{p^{2}-\tilde{m}_{j j}^{2}}{\left(p^{2}-m_{\pi}^{2}\right)^{2}}, \quad \text { for } N_{f}=2 \\
=\frac{i}{p^{2}-m_{\pi}^{2}}-\frac{2 i}{3} \frac{\left(p^{2}-\tilde{m}_{j j}^{2}\right)\left(p^{2}-\tilde{m}_{r r}^{2}\right)}{\left(p^{2}-m_{\pi}^{2}\right)^{2}\left(p^{2}-\tilde{m}_{\eta}^{2}\right)} \\
\quad \text { for } N_{f}=2+1
\end{gathered}
$$

where $\tilde{m}_{\eta}^{2}=\frac{1}{3}\left(\tilde{m}_{j j}^{2}+2 \tilde{m}_{r r}^{2}\right)$.

\section{CALCULATION OF THE $I=2$ PION SCATTERING AMPLITUDE}

Our goal in this work is to calculate the $I=2 \pi \pi$ scattering length in chiral perturbation theory for a partially quenched, mixed action theory with $\mathrm{GW}$ valence quarks and staggered sea quarks, in order to allow correct continuum and chiral extrapolation of mixed action lattice data. We begin, however, by reviewing the pion scattering amplitude in continuum $S U(2)$ chiral perturbation theory. We next calculate the scattering amplitude in $N_{f}=2$ $\mathrm{PQ} \chi \mathrm{PT}$ and MA $\chi \mathrm{PT}$, and finally in $N_{f}=2+1 \mathrm{PQ} \chi \mathrm{PT}$ and $\mathrm{MA} \chi \mathrm{PT}$. When renormalizing divergent 1-loop integrals, we use dimensional regularization and a modified minimal subtraction scheme where we consistently subtract all terms proportional to [41]:

$$
\frac{2}{4-d}-\gamma_{E}+\log 4 \pi+1,
$$

where $d$ is the number of space-time dimensions. The scattering amplitude can be related to the scattering length and other scattering parameters, as we discuss in Sec. V.

\section{A. Continuum $S U(2)$}

The tree-level $I=2$ pion scattering amplitude at threshold is well known to be [40]

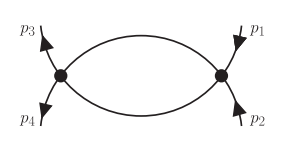

(a)

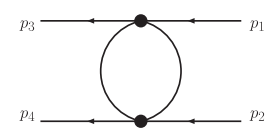

(b)

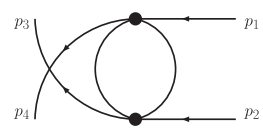

(c)

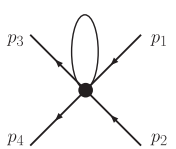

(d)

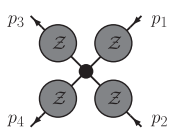

(e)
FIG. 1. One-loop diagrams contributing to the $\pi \pi$ scattering amplitude. Diagrams (a)-(c) are the $s$-, $t$-, and $u$-channel diagrams, respectively, while diagram (e) represents wavefunction renormalization.

$$
i \mathcal{A}=-\frac{4 i m_{\pi}^{2}}{f_{\pi}^{2}}
$$

It is corrected at $\mathcal{O}\left(\varepsilon^{4}\right)$ by loop diagrams and also by treelevel terms from the NLO (or Gasser-Leutwyler) chiral Lagrangian [41]. ${ }^{12}$ The diagrams that contribute at oneloop order are shown in Fig. 1; they lead to the following NLO expression for the scattering amplitude:

$$
\begin{aligned}
i \mathcal{A}_{\vec{p}_{i}=0}= & -\frac{4 i m_{u u}^{2}}{f^{2}}\left\{1+\frac{m_{u u}^{2}}{(4 \pi f)^{2}}\left[8 \ln \left(\frac{m_{u u}^{2}}{\mu^{2}}\right)-1\right.\right. \\
& \left.\left.+l_{\pi \pi}^{\prime}(\mu)\right]\right\},
\end{aligned}
$$

where $m_{u u}$ is the tree-level expression given in Eq. (15) and $f$ is the LO pion decay constant which appears in Eq. (7). The coefficient $l_{\pi \pi}^{\prime}$ is a linear combination of low energy constants appearing in the Gasser-Leutwyler Lagrangian whose scale dependence exactly cancels the scale dependence of the logarithmic term. One can re-express the amplitude, however, in terms of the physical pion mass and decay constant using the NLO formulae for $m_{\pi}$ and $f_{\pi}$ to find:

$$
\begin{aligned}
i \mathcal{A}_{\vec{p}_{i}=0}= & -\frac{4 i m_{\pi}^{2}}{f_{\pi}^{2}}\left\{1+\frac{m_{\pi}^{2}}{\left(4 \pi f_{\pi}\right)^{2}}\left[3 \ln \left(\frac{m_{\pi}^{2}}{\mu^{2}}\right)-1\right.\right. \\
& \left.\left.+l_{\pi \pi}(\mu)\right]\right\},
\end{aligned}
$$

where $l_{\pi \pi}$ is a different linear combination of low energy constants. The expression for $l_{\pi \pi}$ can be found in Ref. [45]. We do not, however, include it here because we do not envision either using the known values of the GasserLeutwyler parameters in the fit of the scattering length or using the fit to determine them. The simple expression (28) has already been used in extrapolation of lattice data from mixed action simulations [29], but it neglects lattice spacing effects from the staggered sea quarks which are known from other simulations to be of the same order as the

\footnotetext{
${ }^{12}$ The continuum $\pi \pi$ scattering amplitude is known to twoloops [43-46].
} 
leading order terms in the chiral expansion of some observables [5]. We therefore proceed to calculate the scattering amplitude in a partially quenched, mixed action theory relevant to simulations.

\section{B. Mixed GW-staggered theory with two sea quarks}

The scattering amplitude in the partially quenched theory differs from the unquenched theory in three important respects. First, more mesons propagate in the loop diagrams. Second, some of the mesons have more complicated propagators due to hairpin diagrams at the quark level [51,55]. Third, there are additional terms in the NLO Lagrangian which arise from partial quenching [52], and lattice spacing effects [10,38].

At the level of quark flow, there are diagrams such as Fig. 2, which route the valence quarks through the diagram in a way which has no ghostly counterpart. Consequently, the ghosts do not exactly cancel the valence quarks in loops. Of course, this is simply a reflection of the fact that the initial and final states, valence pions, are themselves not symmetric under the interchange of ghost and valence quarks, and therefore the graded symmetry between the valence and ghost pions has already been violated. This is well known in quenched and partially quenched heavy baryon $\chi \mathrm{PT}$ [71-73]. This fact also partly explains the success of quenched $\pi \pi$ scattering in the $I=$ 2 channel [30,31]; quenching does not eliminate all loop graphs like it does in many other processes, and, in particular, the $s$-channel diagram is not modified by (partial) quenching effects. As a consequence, it is necessary to compute all the graphs contributing to this process in order to determine the scattering amplitude.

Quark level disconnected (hairpin) diagrams lead to higher order poles in the propagator of any particle which has the quantum numbers of the vacuum $[51,55]$. In the isospin limit of the $N_{f}=2$ partially quenched theory, conservation of isospin prevents the $\pi^{0}$ from suffering any hairpin effects. Hence only the $\bar{\eta}$ acquires a disconnected propagator. Moreover, in the $m_{0} \rightarrow \infty$ limit, the $\bar{\eta}$ propagator (given for a general PQ theory in Eq. (23)) is

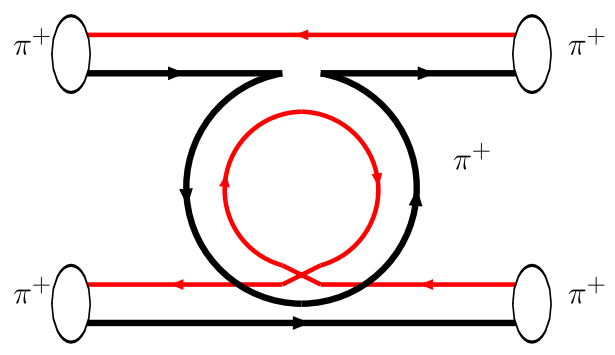

FIG. 2 (color online). Example quark flow for a one-loop $t$-channel graph. This diagram illustrates the presence of meson loops composed of purely valence-valence mesons which are not canceled by valence-ghost loops. Different colors (shades of gray) represent different quark flavors.
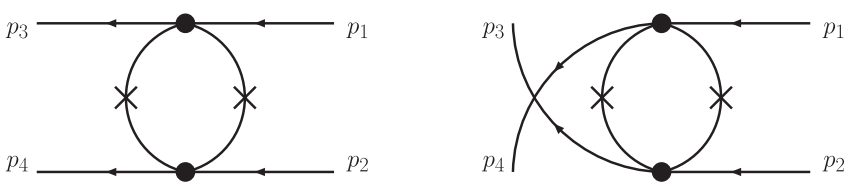

FIG. 3. Example hairpin diagrams contributing to pion scattering. The propagator with a cross through it indicates the quark-disconnected piece of the $\bar{\eta}$ propagator, Eq. (29).

given by the simple expression

$$
\begin{aligned}
G_{\bar{\eta}}\left(p^{2}\right) & =\frac{i}{p^{2}-m_{\pi}^{2}}-i \frac{p^{2}-\tilde{m}_{j j}^{2}}{\left(p^{2}-m_{\pi}^{2}\right)^{2}} \\
& =\frac{i \tilde{\Delta}_{\mathrm{PQ}}^{2}}{\left(p^{2}-m_{\pi}^{2}\right)^{2}},
\end{aligned}
$$

where the parameter

$$
\tilde{\Delta}_{\mathrm{PQ}}^{2}=\tilde{m}_{j j}^{2}-m_{\pi}^{2}
$$

quantifies the partial quenching. (Recall that $\tilde{m}_{j j}$ is the physical mass of a taste singlet sea-sea meson.) Notice that when $\tilde{\Delta}_{\mathrm{PQ}} \rightarrow 0$ the propagator (29) also goes to zero; this is what we expect since, in the $S U(2)$ theory, the only neutral propagating state is the $\pi^{0}$. The propagator in Eq. (29) can appear in loops, thereby producing new diagrams such as those in Fig. 3. ${ }^{13}$ After adding all such hairpin diagrams, one finds that the contribution of the $\bar{\eta}$ to the amplitude is ${ }^{14}$

$$
i \mathcal{A}_{\bar{\eta}}=\frac{4 i}{\left(4 \pi f_{\pi}\right)^{2}} \frac{\tilde{\Delta}_{\mathrm{PQ}}^{4}}{6 f_{\pi}^{2}} \text {. }
$$

In addition to 1-loop contributions, the NLO scattering amplitude receives tree-level analytic contributions from operators of $\mathcal{O}\left(\epsilon^{4}\right)$ in the chiral Lagrangian. At this order, the mixed action Lagrangian contains the same $\mathcal{O}\left(p^{4}\right)$, $\mathcal{O}\left(p^{2} m_{q}\right)$, and $\mathcal{O}\left(m_{q}^{2}\right)$ operators as in the continuum partially quenched chiral Lagrangian, plus additional $\mathcal{O}\left(a^{4}\right)$, $\mathcal{O}\left(a^{2} m_{q}\right)$, and $\mathcal{O}\left(a^{2} p^{2}\right)$ operators arising from discretiza-

\footnotetext{
${ }^{13}$ We note that there are also similar contributions to the four particle vertex with a loop and to the mass correction. We do not show them, however, because they cancel against one another in the amplitude expressed in lattice-physical parameters, which we will show in the following pages.

${ }^{14}$ We note that this contribution does not vanish in the limit that $m_{\pi}^{2} \rightarrow 0$ with $\tilde{m}_{j j}^{2} \neq 0$. Similar effects have been observed in quenched computations of pion scattering amplitudes $[59,60]$. This nonvanishing contribution is the $I=2$ remnant of the divergences that are known to occur in the $I=0$ amplitude at threshold. These divergences give rise to enhanced volume corrections to the $I=0$ amplitude with respect to the one-loop $I=2$ amplitude and prevent the use of Lüscher's formula. Moreover, it is known $[48,50]$ that $\mathrm{PQ} \chi \mathrm{PT}$ is singular in the limit $m_{u} \rightarrow 0$ with nonzero sea quark masses, so the behavior of the amplitude in this limit is meaningless.
} 
tion effects. We can now enumerate the generic forms of analytic contributions from these NLO operators. Because of the chiral symmetry of the GW valence sector, all treelevel contributions to the scattering length must vanish in the limit of vanishing valence quark mass. ${ }^{15}$ Thus there are only three possible forms, each of which must be multiplied by an undetermined coefficient: $m_{u u}^{4}, m_{u u}^{2} m_{j j}^{2}$, and $m_{u u}^{2} a^{2}$. It may, at first, seem surprising that operators of $\mathcal{O}\left(a^{2} m_{q}\right)$, which come from taste-symmetry breaking and contain projectors onto the sea sector, can contribute at tree-level to a purely valence quantity. Nevertheless, this turns out to be the case. These $\mathcal{O}\left(a^{2} m_{q}\right)$ mixed action operators can be determined by first starting with the NLO staggered chiral Lagrangian [10], and then inserting a sea projector, $\mathcal{P}_{S}$, next to every taste matrix. One example of such an operator is $\left[\operatorname{str}\left(\Sigma m_{Q}^{\dagger}\right) \operatorname{str}\left(\mathcal{P}_{S} \xi_{5} \Sigma \xi_{5} \Sigma^{\dagger}\right)+\right.$ p.c.], where, $\xi_{5}$ is the $\gamma_{5}$ matrix acting in taste-space and p.c. indicates parity-conjugate. This double-trace operator will contribute to the lattice pion mass, decay constant, and 4-point function at tree-level because one can place all of the valence pions inside the first supertrace, and the second supertrace containing the projector $\mathcal{P}_{S}$ will just reduce to the identity.

Putting everything together, the total mixed action scattering amplitude to NLO is

$$
\begin{aligned}
i \mathcal{A}_{\vec{p}_{i}=0}= & -\frac{4 i m_{u u}^{2}}{f^{2}}\left\{1+\frac{m_{u u}^{2}}{(4 \pi f)^{2}}\left[4 \ln \left(\frac{m_{u u}^{2}}{\mu^{2}}\right)\right.\right. \\
& \left.+4 \frac{\tilde{m}_{j u}^{2}}{m_{u u}^{2}} \ln \left(\frac{\tilde{m}_{j u}^{2}}{\mu^{2}}\right)-1+l_{\pi \pi}^{\prime}(\mu)\right] \\
& -\frac{m_{u u}^{2}}{(4 \pi f)^{2}}\left[\frac{\tilde{\Delta}_{\mathrm{PQ}}^{4}}{6 m_{u u}^{4}}+\frac{\tilde{\Delta}_{\mathrm{PQ}}^{2}}{m_{u u}^{2}}\left[\ln \left(\frac{m_{u u}^{2}}{\mu^{2}}\right)+1\right]\right] \\
& \left.+\frac{\tilde{\Delta}_{\mathrm{PQ}}^{2}}{(4 \pi f)^{2}} l_{\mathrm{PQ}}^{\prime}(\mu)+\frac{a^{2}}{(4 \pi f)^{2}} l_{a^{2}}^{\prime}(\mu)\right\} .
\end{aligned}
$$

The first two lines of Eq. (32) contain those terms which remain in the continuum and full QCD limit, Eq. (27), while the last two lines account for the effects of partial quenching and of the nonzero lattice spacing. Note that, for consistency with the 1-loop terms, we chose to re-express the analytic contribution proportional to the sea quark mass as $m_{u u}^{2} \tilde{\Delta}_{\mathrm{PQ}}^{2}$. In Eq. (32) we have multiplied every contribution from diagrams which contain a sea quark loop by $1 / 4$, thus making our expression applicable to lattice simulations in which the fourth root of the staggered sea quark determinant is taken.

It is useful, however, to re-express the scattering amplitude in terms of the quantities that one measures in a lattice simulation: $m_{\pi}$ and $f_{\pi}$. Throughout this paper, we will refer to these renormalized measured quantities as the

\footnotetext{
${ }^{15}$ As we discussed in the previous footnote, this condition need not hold for loop contributions to the scattering amplitude.
}

lattice-physical pion mass and decay constant. ${ }^{16}$ Because we are working consistently to second order in chiral perturbation theory, we can equate the lattice-physical pion mass to the 1-loop chiral perturbation theory expression for the pion mass, and likewise for the lattice-physical decay constant. Thus, in terms of lattice-physical parameters, the mixed action $I=2 \pi \pi$ scattering amplitude is

$$
\begin{aligned}
i \mathcal{A}_{\vec{p}_{i}=0}^{\mathrm{MA} \chi \mathrm{PT}}= & -\frac{4 i m_{\pi}^{2}}{f_{\pi}^{2}}\left\{1+\frac{m_{\pi}^{2}}{\left(4 \pi f_{\pi}\right)^{2}}\left[3 \ln \left(\frac{m_{\pi}^{2}}{\mu^{2}}\right)-1\right.\right. \\
& \left.\left.+l_{\pi \pi}(\mu)\right]-\frac{m_{\pi}^{2}}{\left(4 \pi f_{\pi}\right)^{2}} \frac{\tilde{\Delta}_{\mathrm{PQ}}^{4}}{6 m_{\pi}^{4}}\right\}
\end{aligned}
$$

where the first few terms are identical in form to the full QCD amplitude, Eq. (28). This expression for the scattering amplitude is vastly simpler than the one in terms of the bare parameters. First, the hairpin contributions from all diagrams except those in Fig. 3 have exactly cancelled, removing the enhanced chiral logs and leaving the last term in Eq. (33) as the only explicit modification arising from the partial quenching and discretization effects. Second, all contributions from mixed valence-sea mesons in loops have cancelled, thereby removing the new mixed action parameter, $C_{\mathrm{Mix}}$, completely. ${ }^{17}$ Third, all tree-level contributions proportional to the sea quark mass have also cancelled from this expression. And finally, most striking is the fact that an explicit computation of the $\mathcal{O}\left(a^{2} m_{q}\right)$ contributions to the amplitude arising from the NLO mixed action Lagrangian show that these effects exactly cancel when the amplitude is expressed in lattice-physical parameters. This result will be discussed in detail in Ref. [74]. Thus to reiterate, the only partial quenching and lattice spacing dependence in the amplitude comes from the hairpin diagrams of Fig. 3, which produce contributions proportional to $\tilde{\Delta}_{\mathrm{PQ}}^{4}=\left(m_{j j}^{2}+a^{2} \Delta\left(\xi_{I}\right)-m_{\pi}^{2}\right)^{2}$, where $m_{j j}^{2}+a^{2} \Delta\left(\xi_{1}\right)$ is the mass-squared of the tastesinglet sea-sea meson. Moreover, we presume that anyone performing a mixed action lattice simulation will separately measure the taste-singlet sea-sea meson mass and use it as an input to fits of other quantities such as the $\pi \pi$ scattering length. Thus we do not consider it to be an undetermined parameter.

It is now clear that one should fit $\pi \pi$ scattering lattice data in terms of the lattice-physical pion mass and decay constant rather than in terms of the LO pion mass and LO

\footnotetext{
${ }^{16}$ Notice that once the lattice spacing $a$ has been determined, the lattice-physical pion mass can be unambiguously determined by measuring the exponential decay of a pion-pion correlator. We assume that the lattice spacing $a$ has been determined, for example, by studying the heavy quark potential or quarkonium spectrum.

${ }^{17}$ Another consequence of the exact cancelation of the loops with mixed valence-sea quarks is that one does not have to implement the "fourth-root trick" through this order.
} 
decay constant. By doing this, one eliminates three undetermined fit parameters: $C_{\mathrm{Mix}}, l_{\mathrm{PQ}}^{\prime}$, and $l_{a^{2}}^{\prime}$, as well as the enhanced chiral logs.

\section{Mixed GW-staggered theory with $2+1$ sea quarks}

The $2+1$ flavor theory has three additional quarks, the strange valence and ghost and strange sea quarks, which can lead to new contributions to the scattering amplitude. Because we only consider the scattering of valence pions, however, strange valence quarks cannot appear in this process. Thus all new contributions to the scattering amplitude necessarily come only from the sea strange quark, $r$. Because the $r$ quark is heavier than the other sea quarks there is $S U(3)$ symmetry breaking in the sea. This symmetry breaking only affects the pion scattering amplitude, expressed in lattice-physical quantities, through the graphs with internal $\bar{\eta}$ propagators because the masses of the mixed valence-sea mesons cancel in the final amplitude as they did in the earlier two flavor case. In addition, the only signature of partial quenching in the amplitude comes from these same diagrams. It is therefore worthwhile to investigate the physics of the neutral meson propagators further.

There are more hairpin graphs in the $2+1$ flavor theory since the $\eta_{s}$ may propagate as well as the $\eta_{u}$ and the $\eta_{d}$. Because these mesons mix with one another, the flavor basis is not the most convenient basis for the computation. Rather, a useful basis of states is $\pi^{0}, \bar{\eta}=\left(\eta_{u}+\eta_{d}\right) / \sqrt{2}$ and $\eta_{s}$. Since we work in the isospin limit, the $\pi^{0}$ cannot mix with $\bar{\eta}$ or $\eta_{s}$; in addition, there is no vertex between the $\eta_{s}$ and $\pi^{+} \pi^{-}$at this order, so we never encounter a propagating $\eta_{s}$. Thus all the PQ effects are absorbed into the $\bar{\eta}$ propagator, which is given by

$$
G_{\bar{\eta}}\left(p^{2}\right)=\frac{i}{p^{2}-m_{\pi}^{2}}-\frac{2 i}{3} \frac{\left(p^{2}-\tilde{m}_{j j}^{2}\right)\left(p^{2}-\tilde{m}_{r r}^{2}\right)}{\left(p^{2}-m_{\pi}^{2}\right)^{2}\left(p^{2}-\tilde{m}_{\eta}^{2}\right)} .
$$

In $S U$ (3) chiral perturbation theory, the neutral mesons are the $\pi^{0}$ and the $\eta_{8}$. Therefore, in the PQ theory, we know that there will be a contribution from the $\bar{\eta}$ graphs that does not result from partial quenching or $S U(3)$ symmetry breaking. Therefore the extra PQ graphs arising from the internal $\bar{\eta}$ fields must not vanish in the $\tilde{\Delta}_{\mathrm{PQ}} \rightarrow 0$ limit, in contrast to the two flavor case of Eq. (31).

To make this clear, we can re-express the propagator of Eq. (34) in terms of $\tilde{\Delta}_{\mathrm{PQ}}$ as

$G_{\bar{\eta}}\left(p^{2}\right)=i\left[\frac{\tilde{\Delta}_{\mathrm{PQ}}^{2}}{\left(p^{2}-m_{\pi}^{2}\right)^{2}}+\frac{1}{3} \frac{1}{p^{2}-\tilde{m}_{\eta}^{2}}\left(1-\frac{\tilde{\Delta}_{\mathrm{PQ}}^{2}}{p^{2}-m_{\pi}^{2}}\right)^{2}\right]$

This propagator has a single pole which is independent of
$\tilde{\Delta}_{\mathrm{PQ}}$, as well as higher order poles that are at least quadratic in $\tilde{\Delta}_{\mathrm{PQ}}$. It is interesting to consider the large $m_{r}$ limit of this propagator. In this limit, $\tilde{m}_{\eta}^{2} \approx \frac{4}{3} B m_{r}$ is also large. For momenta that are small compared to $\tilde{m}_{\eta}$, the second term of this equation goes to zero in the large $m_{r}$ limit, and the $2+1$ flavor propagator reduces to the 2 flavor propagator, Eq. (29), as expected.

While the above expression clarifies the $\tilde{\Delta}_{\mathrm{PQ}}$ dependence of the propagator and the large $m_{r}$ limit, it obscures the $S U(3)_{\text {sea }}$ limit. An equivalent form of the propagator is

$$
\begin{aligned}
G_{\bar{\eta}}\left(p^{2}\right)= & \frac{i \tilde{\Delta}_{\mathrm{PQ}}^{2}}{\left(p^{2}-m_{\pi}^{2}\right)^{2}} \\
& +\frac{i}{3}\left(1+\frac{\tilde{\Delta}_{3}^{2}}{p^{2}-\tilde{m}_{\eta}^{2}}\right) \frac{1}{p^{2}-m_{\pi}^{2}}\left(1-\frac{\tilde{\Delta}_{\mathrm{PQ}}^{2}}{p^{2}-m_{\pi}^{2}}\right),
\end{aligned}
$$

where the quantity $\tilde{\Delta}_{3}=\sqrt{\tilde{m}_{\eta}^{2}-\tilde{m}_{j j}^{2}}$ parametrizes the $S U(3)_{\text {sea }}$ breaking. When $\tilde{\Delta}_{3}=0$ this propagator is similar in form to the corresponding 2 flavor propagator, Eq. (29), but it has an additional single pole due to the extra neutral meson in the $S U(3)$ theory.

Having considered the new physics of the hairpin propagator, we can now calculate the scattering amplitude. For our purposes here, it is most convenient to express the total $I=2 \pi \pi$ scattering amplitude in terms of $\tilde{\Delta}_{\mathrm{PQ}}$. Just as in the 2-flavor computation, the NLO analytic contributions due to partial quenching and finite lattice spacing effects exactly cancel when the amplitude is expressed in latticephysical parameters. All sea quark mass and lattice spacing dependence comes from the hairpin diagrams, which produce terms proportional to powers of $\tilde{\Delta}_{\mathrm{PQ}}$ with known coefficients. The amplitude is

$$
\begin{aligned}
i \mathcal{A}_{\vec{p}_{i}=0}^{\mathrm{MA} \chi \mathrm{PT}}= & -\frac{4 i m_{\pi}^{2}}{f_{\pi}^{2}}\left\{1+\frac{m_{\pi}^{2}}{\left(4 \pi f_{\pi}\right)^{2}}\left[3 \ln \left(\frac{m_{\pi}^{2}}{\mu^{2}}\right)-1\right.\right. \\
& \left.+\frac{1}{9}\left[\ln \left(\frac{\tilde{m}_{\eta}^{2}}{\mu^{2}}\right)+1\right]+\bar{l}_{\pi \pi}(\mu)\right] \\
& +\frac{1}{\left(4 \pi f_{\pi}\right)^{2}}\left[-\frac{\tilde{\Delta}_{\mathrm{PQ}}^{4}}{6 m_{\pi}^{2}}\right. \\
& \left.\left.+m_{\pi}^{2} \sum_{n=1}^{4}\left(\frac{\tilde{\Delta}_{\mathrm{PQ}}^{2}}{m_{\pi}^{2}}\right)^{n} \mathcal{F}_{n}\left(\tilde{m}_{\eta}^{2} / m_{\pi}^{2}\right)\right]\right\}
\end{aligned}
$$

where $\tilde{\Delta}_{\mathrm{PQ}}^{2}=m_{j j}^{2}+a^{2} \Delta\left(\xi_{I}\right)-m_{\pi}^{2}$ and 


$$
\begin{aligned}
\mathcal{F}_{1}(x)= & -\frac{2}{9(x-1)^{2}}[5(x-1)-(3 x+2) \ln (x)] \\
\mathcal{F}_{2}(x)= & \frac{2}{3(x-1)^{3}}[(x-1)(x+3)-(3 x+1) \ln (x)] \\
\mathcal{F}_{3}(x)= & \frac{1}{9(x-1)^{4}}\left[(x-1)\left(x^{2}-7 x-12\right)\right. \\
& +2(7 x+2) \ln (x)] \\
\mathcal{F}_{4}(x)= & -\frac{1}{54(x-1)^{5}}\left[(x-1)\left(x^{2}-8 x-17\right)\right. \\
& +6(3 x+1) \ln (x)]
\end{aligned}
$$

The functions $\mathcal{F}_{i}$ have the property that $\mathcal{F}_{i}(x) \rightarrow 0$ in the limit that $x \rightarrow \infty$. Therefore, when the strange sea quark mass is very large, i.e. $\tilde{m}_{\eta}^{2} / m_{\pi}^{2} \gg 1$, the $2+1$ flavor amplitude reduces to the 2 flavor amplitude, Eq. (33), with the exception of terms that can be absorbed into the analytic terms. The low energy constants have a scale dependence which exactly cancels the scale dependence in the logarithms. The coefficient $\bar{l}_{\pi \pi}$ is the same linear combination of Gasser-Leutwyler coefficients that appear in the $S U(3)$ scattering amplitude expressed in terms of the physical pion mass and decay constant $[43,46]$.

Because the functions $\mathcal{F}_{i}$ depend logarithmically on $x$, the $2+1$ flavor scattering amplitude features enhanced chiral logarithms [48] that are absent from the 2 flavor amplitude. This is a useful observation, as we will now explain. Because there is a strange quark in nature and its mass is less than the QCD scale, $\Lambda_{\mathrm{QCD}}$, lattice simulations must use $2+1$ quark flavors. It is often practical to fix the strange quark mass at a constant value near its physical value in these simulations. This circumstance is helpful because, just as $S U(2)$ chiral perturbation theory is useful to describe nature at scales smaller than the strange quark mass, the 2 flavor amplitude given in Eq. (33) can be used to extrapolate $2+1$ flavor lattice data at energy scales smaller than the strange sea quark mass used in the simulation (provided, of course, there are no strange valence quarks) [75]. This is valid because, at energy scales smaller than the strange quark mass (or actually twice the strange quark mass, since the purely pionic systems have no valence strange quarks), one can integrate out the strange quark. This is not an approximation, because all of the effects of the strange quark are absorbed into a renormalization of the parameters of the chiral Lagrangian. Moreover, since the 2 flavor amplitude does not exhibit enhanced chiral logarithms, signatures of partial quenching can be reduced by extrapolating lattice data with the 2 flavor, rather than the $2+1$ flavor, expression. We note that in this case the effects of the strange quark are absorbed in the coefficients of the analytic terms appearing in Eq. (33), and thus they are not constant, but rather depend logarithmically upon the strange sea quark mass.

\section{V. $I=2$ PION SCATTERING LENGTH RESULTS}

In this section we present our results for the $s$-wave $I=$ $2 \pi \pi$ scattering length in the two theories most relevant to current mixed action lattice simulations: those with GW valence quarks and either $N_{f}=2$ or $N_{f}=2+1$ staggered sea quarks. We only present results for the scattering length expressed in lattice-physical parameters. The $s$-wave scattering length is trivially related to the full scattering amplitude at threshold by an overall prefactor:

$$
a_{l=0}^{(I=2)}=\left.\frac{1}{32 \pi m_{\pi}} \mathcal{A}^{I=2}\right|_{\vec{p}_{i}=0} .
$$

\section{A. Scattering length with 2 sea quarks}

The $I=2 \pi \pi s$-wave scattering length in a MA $\chi \mathrm{PT}$ theory with 2 sea quarks is given by

$$
\begin{aligned}
a_{0}^{(2) \mathrm{MA} \chi \mathrm{PT}}= & -\frac{m_{\pi}}{8 \pi f_{\pi}^{2}}\left\{1+\frac{m_{\pi}^{2}}{\left(4 \pi f_{\pi}\right)^{2}}\left[3 \ln \left(\frac{m_{\pi}^{2}}{\mu^{2}}\right)-1\right.\right. \\
& \left.\left.+l_{\pi \pi}(\mu)\right]-\frac{m_{\pi}^{2}}{\left(4 \pi f_{\pi}\right)^{2}} \frac{\tilde{\Delta}_{\mathrm{PQ}}^{4}}{6 m_{\pi}^{4}}\right\}
\end{aligned}
$$

where $\tilde{\Delta}_{\mathrm{PQ}}^{2}=m_{j j}^{2}+a^{2} \Delta\left(\xi_{I}\right)-m_{\pi}^{2}$. The first two terms are the result one obtains in $S U(2) \chi \mathrm{PT}$ [45] and the last term is the only new effect arising from the partial quenching and mixed action. All other possible partial quenching terms, enhanced chiral logs and additional linear combinations of the $\mathcal{O}\left(p^{4}\right)$ Gasser-Leutwyler coefficients, exactly cancel when the scattering length is expressed in terms of lattice-physical parameters. And, most strikingly, the pion mass, decay constant and the 4-point function all receive $\mathcal{O}\left(a^{2} m_{q}\right)$ corrections from the lattice, but they exactly cancel in the scattering length expressed in terms of the lattice-physical parameters [74]. It is remarkable that the only artifact of the nonzero lattice spacing, $m_{j j}^{2}+$ $a^{2} \Delta_{I}$, can be separately determined simply by measuring the exponential fall-off of the taste-singlet sea-sea meson 2-point function. Thus there are no undetermined fit parameters in the mixed action scattering length expression from either partial quenching or lattice discretization effects; there is only the unknown continuum coefficient, $l_{\pi \pi}$.

One can trivially deduce the continuum PQ scattering length from Eq. (40): simply let $a \rightarrow 0$, reducing $\tilde{m}_{j j} \rightarrow$ $m_{j j}=2 B m_{j}$ in $\tilde{\Delta}_{\mathrm{PQ}}$, resulting in

$$
\begin{aligned}
a_{0}^{(2) \mathrm{PQ} \chi \mathrm{PT}}= & -\frac{m_{\pi}}{8 \pi f_{\pi}^{2}}\left\{1+\frac{m_{\pi}^{2}}{\left(4 \pi f_{\pi}\right)^{2}}\left[3 \ln \left(\frac{m_{\pi}^{2}}{\mu^{2}}\right)-1\right.\right. \\
& \left.\left.+l_{\pi \pi}(\mu)\right]-\frac{\Delta_{\mathrm{PQ}}^{4}}{6\left(4 \pi m_{\pi} f_{\pi}\right)^{2}}\right\}
\end{aligned}
$$




\section{B. Scattering length with $2+1$ sea quarks}

The $I=2 \pi \pi s$-wave scattering length in a MA $\chi \mathrm{PT}$ theory with $2+1$ sea quarks is given by

$$
\begin{aligned}
a_{0}^{(2) \mathrm{MA} \chi \mathrm{PT}}= & -\frac{m_{\pi}}{8 \pi f_{\pi}^{2}}\left\{1+\frac{m_{\pi}^{2}}{\left(4 \pi f_{\pi}\right)^{2}}\left[3 \ln \left(\frac{m_{\pi}^{2}}{\mu^{2}}\right)-1\right.\right. \\
& \left.+\frac{1}{9}\left[\ln \left(\frac{\tilde{m}_{\eta}^{2}}{\mu^{2}}\right)+1\right]+\bar{l}_{\pi \pi}(\mu)\right] \\
& +\frac{1}{\left(4 \pi f_{\pi}\right)^{2}}\left[-\frac{\tilde{\Delta}_{\mathrm{PQ}}^{4}}{6 m_{\pi}^{2}}\right. \\
& \left.\left.+m_{\pi}^{2} \sum_{n=1}^{4}\left(\frac{\tilde{\Delta}_{\mathrm{PQ}}^{2}}{m_{\pi}^{2}}\right)^{n} \mathcal{F}_{n}\left(\tilde{m}_{\eta}^{2} / m_{\pi}^{2}\right)\right]\right\}
\end{aligned}
$$

where the functions $\mathcal{F}_{i}$ are defined in Eq. (38). As in the 2flavor MA $\chi$ PT expression, Eq. (40), the only undetermined parameter is the linear combination of Gasser-Leutwyler coefficients, $\bar{l}_{\pi \pi}$, which also appears in the continuum $\chi \mathrm{PT}$ expression.

We note as an aside that this suppression of lattice spacing counterterms is in contrast to the larger number of terms that one would need in order to correctly fit data from simulations with Wilson valence quarks on Wilson sea quarks. Because the Wilson action breaks chiral symmetry at $\mathcal{O}(a)$, even for massless quarks, there will be terms proportional to all powers of the lattice spacing in the expression for the scattering length in Wilson $\chi \mathrm{PT}$ $[74,76,77]$. Moreover, such lattice spacing corrections begin at $\mathcal{O}(a)$, rather than $\mathcal{O}\left(a^{2}\right)$. If one uses $\mathcal{O}(a)$ improved Wilson quarks, then the leading discretization effects are of $\mathcal{O}\left(a^{2}\right)$, as for staggered quarks; however, this does not remove the additional chiral symmetry-breaking operators. Another practical issue is whether or not one can perform simulations with Wilson sea quarks that are light enough to be in the chiral regime.

\section{DISCUSSION}

Considerable progress has recently been made in fully dynamical simulations of pion scattering in the $I=2$ channel $[29,33]$. We have considered $I=2$ scattering of pions composed of Ginsparg-Wilson quarks on a staggered sea. We have calculated the scattering length in both this mixed action theory and in continuum $\mathrm{PQ} \chi \mathrm{PT}$ for theories with either 2 or $2+1$ dynamical quarks. These expressions are necessary for the correct continuum and chiral extrapolation of PQ and mixed action lattice data to the physical pion mass.

Our formulae, Eqs. (40) and (42), not only provide the form for the mixed action scattering length, but also contain two predictions relevant to the recent work of Ref. [29]. Beane et. al. calculated the $I=2 s$-wave $\pi \pi$ scattering length using domain-wall valence quarks and staggered sea quarks, but used the continuum $\chi$ PT expres- sion to extrapolate to the physical quark masses. In Fig. 2 of Ref. [29], which plots $m_{\pi} a_{2}^{(0)}$ versus $m_{\pi} / f_{\pi}$, the fit of the $\chi$ PT expression to the lattice data overshoots the lightest pion mass point but fits the heavier two points quite well. This is interesting because Eq. (40) predicts a known, positive shift to $m_{\pi} a_{2}^{(0)}$ of size $\tilde{\Delta}_{\mathrm{PQ}}^{4} /\left(768 f_{\pi}^{4} \pi^{3}\right)$. Accounting for this positive shift is equivalent to lowering the entire curve, and could therefore move the fit such that it goes between the data points. This turns out, however, not to be the case. In Ref. [29], the valence and sea quark masses are tuned to be equal, so $\tilde{\Delta}_{\mathrm{PQ}}^{2}=a^{2} \Delta_{I} \simeq$ $(446 \mathrm{MeV})^{2}$ [5]. Despite the large value of $\tilde{\Delta}_{\mathrm{PQ}}$, the predicted shift is insignificant, being an order of magnitude less than the statistical error. In Table I, we collect the predicted shifts to $m_{\pi} a_{2}^{(0)}$ at the three pion masses used in Ref. [29]. We also list the magnitude of the ratio of these predicted shifts to the leading contribution to the scattering length, which turn out to be small, lending confidence to the power counting we have used, Eq. (11). The other more important prediction is that there are no unknown corrections to the $\chi \mathrm{PT}$ formula for the scattering length arising from lattice spacing corrections or partial quenching through the order $\mathcal{O}\left(m_{q}^{2}\right), \mathcal{O}\left(a^{2} m_{q}\right)$ and $\mathcal{O}\left(a^{4}\right)$. Therefore, to within statistical and systematic errors, the continuum $\chi \mathrm{PT}$ expression used by Beane $e t$. al. to fit their numerical $\pi \pi$ scattering data [29] receives no corrections through the 1-loop level.

The central result of this paper is that the appropriate way to extrapolate lattice $\pi \pi$ scattering data is in terms of the lattice-physical pion mass and decay constant rather than in terms of the LO parameters which appear in the chiral Lagrangian. When expressed in terms of the LO parameters, the scattering length depends upon 4 undetermined parameters, $l_{\pi \pi}^{\prime}, l_{\mathrm{PQ}}^{\prime}, l_{a^{2}}^{\prime}$ and $C_{\mathrm{Mix}}$. In contrast, the scattering length expressed in terms of the lattice-physical parameters depends upon only one unknown parameter, $l_{\pi \pi}$, the same linear combination of Gasser-Leutwyler coefficients which contributes to the scattering length in continuum $\chi \mathrm{PT}$.

TABLE I. Predicted shifts to the scattering length computed in Ref. [29] arising from finite lattice spacing effects in the mixed action theory. The first two rows show the approximate values of $m_{\pi}$ and $f_{\pi}$ while the third shows $m_{\pi} a_{2}^{(0)}$ plus the statistical error calculated in [29]. In the fourth row, we give the predicted shifts in the scattering length (times $m_{\pi}$ ) and, in the fifth row, we give the ratio of the predicted shift to the leading order contribution to the scattering length.

\begin{tabular}{lccc}
\hline \hline$m_{\pi}(\mathrm{MeV})$ & 294 & 348 & 484 \\
$f_{\pi}(\mathrm{MeV})$ & 145 & 149 & 158 \\
$m_{\pi} a_{2}^{(0)}$ & $-0.212 \pm 0.024$ & $-0.222 \pm 0.014$ & $-0.38 \pm 0.03$ \\
$\tilde{\Delta}_{\mathrm{PQ}}^{4} / 768 \pi^{3} f_{\pi}^{4}$ & 0.00374 & 0.00336 & 0.00266 \\
$\tilde{\Delta}_{\mathrm{PQ}}^{4} / 6\left(4 \pi f_{\pi} m_{\pi}\right)^{2}$ & 0.0229 & 0.0155 & 0.00711 \\
\hline \hline
\end{tabular}




\section{ACKNOWLEDGMENTS}

We would like to thank Maarten Golterman, Martin Savage, and Steve Sharpe for many useful discussions and helpful comments on the manuscript. We would also like to thank the referee for helpful comments regarding the manuscript. R. V. would also like to thank Jack Laiho for a helpful discussion and for pointing out a relevant reference. J.W.C. and A. W. L. would like to thank the ECT* and the organizers of Effective theories in Nuclear physics and Lattice QCD, Paulo Bedaque, Elisabetta Pallante, and Assumpta Parreno for organizing a wonderful conference and providing a stimulating atmosphere where we formulated the idea for this project. J. W. C. thanks the INT at the University of Washington for hospitality. D. O.C. thanks the nuclear theory group at the University of Washington for hospitality during the completion of this work. J. W. C. is supported by the National Science Council of R.O.C. D. O.C. is supported in part by the U.S. DOE under the grant No. DE-FG03-9ER40701. R. V. was supported under DOE grant No. DE-FG0296ER40956. A.W.L. is supported under DOE grant No. DE-FG03-97ER41014.
[1] C. Bernard et al., Nucl. Phys. B, Proc. Suppl. 119, 170 (2003).

[2] S. R. Beane, Nucl. Phys. B695, 192 (2004).

[3] L. Susskind, Phys. Rev. D 16, 3031 (1977).

[4] C. T.H. Davies et al. (HPQCD), Phys. Rev. Lett. 92, 022001 (2004).

[5] C. Aubin et al. (MILC), Phys. Rev. D 70, 114501 (2004).

[6] S. Durr, Proc. Sci. LAT2005 (2005) 021.

[7] W.-J. Lee and S.R. Sharpe, Phys. Rev. D 60, 114503 (1999).

[8] C. Aubin and C. Bernard, Phys. Rev. D 68, 034014 (2003).

[9] C. Aubin and C. Bernard, Phys. Rev. D 68, 074011 (2003).

[10] S. R. Sharpe and R. S. Van de Water, Phys. Rev. D 71, 114505 (2005).

[11] R.S. Van de Water and S. R. Sharpe, Phys. Rev. D 73, 014003 (2006).

[12] M.F. L. Golterman and J. Smit, Nucl. Phys. B245, 61 (1984).

[13] M. F. L. Golterman, Nucl. Phys. B273, 663 (1986).

[14] M.F. L. Golterman and J. Smit, Nucl. Phys. B255, 328 (1985).

[15] M.F. L. Golterman, Nucl. Phys. B278, 417 (1986).

[16] J. A. Bailey and C. Bernard, Proc. Sci. LAT2005 (2005) 047.

[17] P. H. Ginsparg and K. G. Wilson, Phys. Rev. D 25, 2649 (1982).

[18] M. Luscher, Phys. Lett. B 428, 342 (1998).

[19] D. B. Kaplan, Phys. Lett. B 288, 342 (1992).

[20] Y. Shamir, Nucl. Phys. B406, 90 (1993).

[21] V. Furman and Y. Shamir, Nucl. Phys. B439, 54 (1995).

[22] R. Narayanan and H. Neuberger, Nucl. Phys. B412, 574 (1994).

[23] R. Narayanan and H. Neuberger, Phys. Rev. Lett. 71, 3251 (1993).

[24] R. Narayanan and H. Neuberger, Nucl. Phys. B443, 305 (1995).

[25] A.D. Kennedy, Nucl. Phys. B, Proc. Suppl. 140, 190 (2005).

[26] D. B. Renner et al. (LHP), Nucl. Phys. B, Proc. Suppl. 140, 255 (2005).

[27] K. C. Bowler, B. Joo, R. D. Kenway, C. M. Maynard, and
R. J. Tweedie (UKQCD), J. High Energy Phys. 08 (2005) 003.

[28] F. D. R. Bonnet, R. G. Edwards, G. T. Fleming, R. Lewis, and D. G. Richards (Lattice Hadron Physics), Phys. Rev. D 72, 054506 (2005).

[29] S. R. Beane, P. F. Bedaque, K. Orginos, and M. J. Savage (NPLQCD), Phys. Rev. D 73, 054503 (2006).

[30] S. R. Sharpe, R. Gupta, and G. W. Kilcup, Nucl. Phys. B383, 309 (1992).

[31] R. Gupta, A. Patel, and S. R. Sharpe, Phys. Rev. D 48, 388 (1993).

[32] S. Aoki et al. (JLQCD), Phys. Rev. D 66, 077501 (2002).

[33] T. Yamazaki et al. (CP-PACS), Phys. Rev. D 70, 074513 (2004).

[34] S. Aoki et al. (CP-PACS), Phys. Rev. D 71, 094504 (2005).

[35] O. Bar, G. Rupak, and N. Shoresh, Phys. Rev. D 67, 114505 (2003).

[36] O. Bar, G. Rupak, and N. Shoresh, Phys. Rev. D 70, 034508 (2004).

[37] B. C. Tiburzi, Nucl. Phys. A 761, 232 (2005).

[38] O. Bar, C. Bernard, G. Rupak, and N. Shoresh, Phys. Rev. D 72, 054502 (2005).

[39] B. C. Tiburzi, Phys. Rev. D 72, 094501 (2005).

[40] S. Weinberg, Phys. Rev. Lett. 17, 616 (1966).

[41] J. Gasser and H. Leutwyler, Ann. Phys. (N.Y.) 158, 142 (1984).

[42] J. Gasser and H. Leutwyler, Nucl. Phys. B250, 465 (1985).

[43] M. Knecht, B. Moussallam, J. Stern, and N.H. Fuchs, Nucl. Phys. B457, 513 (1995).

[44] J. Bijnens, G. Colangelo, G. Ecker, J. Gasser, and M.E. Sainio, Phys. Lett. B 374, 210 (1996).

[45] J. Bijnens, G. Colangelo, G. Ecker, J. Gasser, and M.E. Sainio, Nucl. Phys. B508, 263 (1997).

[46] J. Bijnens, P. Dhonte, and P. Talavera, J. High Energy Phys. 01 (2004) 050.

[47] C. W. Bernard and M.F.L. Golterman, Phys. Rev. D 49, 486 (1994).

[48] S. R. Sharpe, Phys. Rev. D 56, 7052 (1997).

[49] M.F.L. Golterman and K.-C. Leung, Phys. Rev. D 57, 5703 (1998). 
[50] S. R. Sharpe and N. Shoresh, Phys. Rev. D 62, 094503 (2000).

[51] S. R. Sharpe and N. Shoresh, Phys. Rev. D 64, 114510 (2001).

[52] S. R. Sharpe and R. S. Van de Water, Phys. Rev. D 69, 054027 (2004).

[53] M. Luscher, Commun. Math. Phys. 105, 153 (1986).

[54] M. Luscher, Nucl. Phys. B354, 531 (1991).

[55] C. W. Bernard and M. Golterman, Nucl. Phys. B, Proc. Suppl. 34, 331 (1994).

[56] L. Maiani and M. Testa, Phys. Lett. B 245, 585 (1990).

[57] K. Rummukainen and S. A. Gottlieb, Nucl. Phys. B450, 397 (1995).

[58] C. H. Kim, C. T. Sachrajda, and S. R. Sharpe, Nucl. Phys. B727, 218 (2005).

[59] C. W. Bernard and M.F.L. Golterman, Phys. Rev. D 53, 476 (1996).

[60] G. Colangelo and E. Pallante, Nucl. Phys. B520, 433 (1998).

[61] C. J. D. Lin, G. Martinelli, E. Pallante, C. T. Sachrajda, and G. Villadoro, Phys. Lett. B 553, 229 (2003).

[62] C. J. D. Lin, G. Martinelli, E. Pallante, C. T. Sachrajda, and G. Villadoro, Phys. Lett. B 581, 207 (2004).

[63] S. R. Beane and M. J. Savage, Phys. Rev. D 67, 054502 (2003).

[64] M. Golterman, T. Izubuchi, and Y. Shamir, Phys. Rev. D
71, 114508 (2005).

[65] C. J. D. Lin, G. Martinelli, E. Pallante, C. T. Sachrajda, and G. Villadoro, Nucl. Phys. B650, 301 (2003).

[66] S. R. Beane, P. F. Bedaque, A. Parreno, and M. J. Savage, Phys. Lett. B 585, 106 (2004).

[67] P.F. Bedaque and J.-W. Chen, Phys. Lett. B 616, 208 (2005).

[68] S. R. Sharpe and J. Singleton Robert, Phys. Rev. D 58, 074501 (1998).

[69] K. Symanzik, Nucl. Phys. B226, 205 (1983).

[70] K. Symanzik, Nucl. Phys. B226, 187 (1983).

[71] J. N. Labrenz and S. R. Sharpe, Phys. Rev. D 54, 4595 (1996).

[72] J.-W. Chen and M.J. Savage, Phys. Rev. D 65, 094001 (2002).

[73] S. R. Beane and M. J. Savage, Nucl. Phys. A 709, 319 (2002).

[74] J.-W. Chen, D. O'Connell, and A. Walker-Loud (to be published).

[75] J.-W. Chen and M. J. Savage, Phys. Rev. D 66, 074509 (2002).

[76] G. Rupak and N. Shoresh, Phys. Rev. D 66, 054503 (2002).

[77] S. Aoki, O. Bar, S. Takeda, and T. Ishikawa, Phys. Rev. D 73, 014511 (2006). 